\title{
Dinâmica de participação social na construção coletiva de informação no campo museal: estudo de caso dos museus na Wikipédia no âmbito do Instituto Brasileiro de Museus
}

Dynamics of social participation in the collective construction of information in the museum field: case study of the museums in Wikipedia under the Brazilian Institute of Museums

\author{
Dalton Lopes Martins* \\ Danielle do Carmo*
}

\section{RESUMO}

O presente artigo identifica e descreve dados relativos à dinâmica de participação coletiva e colaborativa na produção de informações sobre os museus brasileiros na Wikipédia. Utilizando como amostra os museus administrados diretamente pelo Instituto Brasileiro de Museus (Ibram), foi realizada a coleta de dados da Wikipédia utilizando o software XTools. Dessa forma o presente artigo busca demonstrar o potencial da Wikipédia como fonte de pesquisa, principalmente no que tange à produção de informações sobre os museus brasileiros na internet. Os resultados identificam que 20 museus possuem página na Wikipédia, tendo sido editadas por um total de 555 usuários que realizaram um total de 1.108 edições nas páginas. Identifica-se grande desigualdade na dinâmica de produção de informação nas páginas e uma expressiva rede de editores realizando ações de curadoria.

Palavras-chave: Wikipédia; Museus

\begin{abstract}
This article identifies and describes data on the dynamics of collective and collaborative participation in the production of information about Brazilian museums on Wikipedia. Using as sample the museums administered directly by the Brazilian Institute of Museums (Ibram), the data gathering was carried out articles using the XTools software. In this way, the present article seeks to demonstrate the potential of Wikipedia as a source of research, especially in what concerns the production of information about Brazilian museums on the Internet. The results identify that 20 museums have a page on Wikipedia, having been edited by a total of 555 users who have made a total of 1.108 editions on the pages. It identifies great inequality in the dynamics of information production in the pages and an expressive network of publishers performing curatorial actions.
\end{abstract}

Keywords: Wikipedia; Brazilian Museums; Source of Information; Digital

\footnotetext{
* Doutor em Ciência da Informação pela Universidade de São Paulo. Professor do curso de graduação de Biblioteconomia e do Programa de Pós-Graduação em Ciência da Informação da Universidade de Brasília (UnB). Endereço: Campus Universitário Darcy Ribeiro, Faculdade de Ciência da Informação, Edifício da Biblioteca Central, Entrada Leste, CEP: 70.910-900, Brasília, DF. Telefone: (61) 3107-2632. E-mail: daltonmartins@unb.br

** Mestre em Memória Social e Patrimônio Cultural pela Universidade Federal de Pelotas (UFPel) e pesquisadora do projeto Tainacan - Acervos Digitais em Rede da Universidade Federal de Goiás (UFG). Endereço: Rua Samambaia, Campus Samambaia, UFG, CEP 74.690-900, Goiânia,GO. Telefone: (62) 35211659. E-mail: docarmo.danielle@gmail.com.
} 
Brasileiros; Fonte de Informação; Humanities; Ibram. Humanidades Digitais; Ibram.

\section{INTRODUÇÃO}

O surgimento e a popularização dos dispositivos digitais, nas quais as tecnologias de comunicação e informação contemporâneas estão baseadas, acabaram por alterar de forma radical os processos de aquisição e produção de informação e conhecimento, sobretudo nas últimas duas décadas. As plataformas de busca online, como o Google, as redes sociais, como o Facebook, e os aplicativos de mensagens instantâneas, como o WhatsApp, parecem se tornar os protagonistas nos processos cotidianos de comunicação e busca por informações.

Da mesma forma que as tecnologias digitais alteraram significativamente os processos cotidianos, também impactaram o campo científico de forma substancial. Como consequência da gradual incorporação das tecnologias digitais nos processos e procedimento de pesquisas, surgiram novos temas, objetos, práticas, abordagens, métodos e ferramentas.

No âmbito das ciências humanas e sociais, o conjunto de práticas científicas que adotam as tecnologias digitais em suas abordagens e/ou procedimentos encontra-se sob o termo "humanidades digitais". Em relação ao desenvolvimento de pesquisa no campo das humanidades digitais, Berry (2011) observa que:

[...] a primeira onda das humanidades digitais envolvia a construção de infraestrutura nos estudos dos textos de humanidades através dos repositórios digitais, anotações de texto e etc., enquanto a segunda onda das humanidades digitais expande os limites das noções de arquivo para incluir obras digitais e assim trazem à tona, para as humanidades, seus próprios conjuntos de ferramentas para examinar materiais "nascidos digitais", como a literatura eletrônica (e-lit), ficção interativa (IF), artefatos baseados na web e assim por diante (BERRY, 2011, p. 3-4, tradução nossa).

Pode-se dizer que o presente estudo reflete a segunda onda das humanidades digitais descrita por Berry (2011), uma vez que busca realizar uma investigação com base nos dados, "nascidos digitais", de um artefato baseado na web, a Wikipédia, por meio de uma ferramenta digital, a XTools, tendo como objetivos identificar e descrever aspectos da dinâmica da produção coletiva e colaborativa de informações sobre os museus brasileiros, mais especificamente os que se encontram sob administração direta do Instituto Brasileiros de Museus (Ibram).

Dessa forma, no âmbito do presente artigo, apresentaremos, em um primeiro momento, a Wikipédia por meio do diálogo com os referenciais bibliográficos, suas principais características de produção, e uma visão geral da presença de verbetes sobre os museus brasileiros. Em um segundo momento, será apresentada a metodologia utilizada para coleta de dados. Por meio da análise desses dados, os resultados serão posteriormente descritos. 


\section{A Wikipédia como fonte de informação}

O trabalho enciclopédico é caracterizado pela ambição de reunir e expor, de forma ordenada e concisa, a soma do conhecimento humano sobre diferentes domínios do conhecimento. Segundo Campello (2008), a ocorrência de obras resultantes do trabalho enciclopédico pode ser observada desde a Antiguidade, entretanto a autora localiza o emprego do termo "enciclopédia", nos moldes que conhecemos, somente no século XVI. Ao longo do tempo, a concepção do que seria uma enciclopédia foi se modificando e evoluindo de acordo com "as necessidades culturais e educacionais da sociedade" (CAMPELLO, 2008, p.9).

Com a popularização e desenvolvimento das tecnologias digitais, a Wikipédia surge como um novo formato enciclopédico, que, segundo Peter Burke (2002), diferenciase das enciclopédias convencionais em vários aspectos, entre eles o tamanho, a disponibilidade em diversos idiomas, o constante processo de revisão e reconstrução, a produção do conteúdo por seus leitores e a possibilidade de uma rápida recuperação da informação.

Atualmente a Wikipédia configura-se como uma das principais fontes de informação enciclopédica da web, sendo o $5^{\circ}$ site mais visitado do mundo, segundo o ranking da Alexa Internet ${ }^{1}$ (2018), ficando atrás do mecanismo de busca chinês Baidu (4 $4^{\circ}$ ), da rede social Facebook $\left(3^{\circ}\right)$, da rede de vídeos YouTube $\left(2^{\circ}\right)$ e do mecanismo de busca Google $\left(1^{\circ}\right)$.

Lançada formalmente no dia 15 de janeiro de 2001, a Wikipédia utiliza um software tipo wiki, o acesso ao seu conteúdo é gratuito, e a licença é aberta (licença Creative Commons Attribution-ShareAlike e GNU). Devido à sua proposta multilíngue, atualmente existem versões da Wikipédia em 303 idiomas. $^{2}$ Em 2018, a Wikipédia

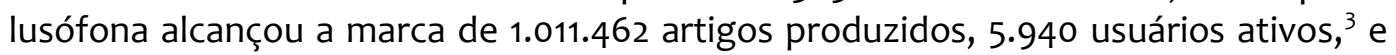
atinge uma média diária de 9.792 .026 visualizações. ${ }^{4}$

A Wikipédia é um dos projetos ${ }^{5}$ sob gestão da Fundação Wikimedia, organização sem fins lucrativo sediada nos Estados Unidos da América que declara como missão "empoderar e engajar pessoas pelo mundo para coletar e desenvolver conteúdo educacional sob uma licença livre ou no domínio público, e para disseminá-lo efetivamente e globalmente" (WIKIMEDIA FOUNDATION, 2018).

Apesar de ser um dos sites mais acessados da web, existe uma intensa discussão acerca da qualidade de suas informações no meio acadêmico. Os autores Medeiros e Sousa (2018, p.74) relatam a percepção de que no âmbito da ciência da informação e da biblioteconomia, principalmente em sua bibliografia, a enciclopédia ainda é

\footnotetext{
1 A Alexa Internet é uma companhia que oferece dados de tráfego na internet e análise de dados (Wikipédia). Dados coletados dia 19 de janeiro de 2019.

2 Destes, 293 ativos. Lista das instalações disponível em: <https://meta.wikimedia.org/wiki/List_of_Wikipedias>. Acesso: 9 jan. 2019.

3 Dados coletados dia 4 de dez. 2018. Dados disponíveis em: <https://pt.wikipedia.org/wiki/Wikip\%C3\%Agdia:P\%C3\%A1gina_principal>. Acesso: 4 dez. 2018.

$4 \quad$ Disponível em: <https://tools.wmflabs.org/siteviews/?platform=allaccess\&source=pageviews\&agent=user\&range=latest-20\&sites=pt.wikipedia.org $>$. Acesso em: 19 jan. 2019.

${ }^{5}$ Além da Wikipédia, a Fundação Wikimedia também desenvolve projetos como a Wikidata, Wikimedia Commons, Wikcionário, Wikisource, entre outros.
} 
considerada como fonte de informação quando apresentada em seu formato clássico. Digitais ou impressas, as enciclopédias em seu modo de produção tradicional - elaboradas e revisadas por especialistas - permitiria que características como autoridade e confiabilidade fossem claramente definidas. Entretanto, os autores ressaltam que a expansão da Wikipédia nas últimas décadas e os esforços de especialistas e não especialistas em sua produção deveriam ser considerados em análises que tomam as enciclopédias como fonte de informação devido à relevância que apresenta. Dessa forma, os autores classificam a Wikipédia como uma fonte de informação terciária, "uma vez que se coloca como uma espécie de guia sobre conhecimento estabelecido" (MEDEIROS; SOUZA, 2018, p.74).

Segundo Kern (2018), as avaliações sobre a qualidade da Wikipédia começaram a ganhar força quatro anos depois de sua publicação, quando a revista Nature (GILES, 2005) realizou uma pesquisa comparando-a com a Encyclopedia Britannica. O estudo acabou por apontar a superioridade da Wikipédia. Após esse estudo, outras pesquisas no âmbito internacional (HORLAND, 2011; MESGARI et al., 2015; STIVILIA el al., 2008) e nacional (D'ANDREA, 2009), utilizando métodos e critérios de comparação distintos, acabaram por concluir que a Wikipédia apresenta vantagens em relação as outras enciclopédias.

Em resumo, a literatura internacional parece convergir para o que concluem Arazy et al. (2011): que o sucesso da Wikipédia mostra que é possível comunidades auto-organizadas construírem produtos informacionais de alta qualidade. As análises da Wikipédia são cada vez mais detalhadas e críticas, sem espaço para elogios ou rejeições simplistas. A literatura brasileira em ciência da informação está em acordo com os estudos internacionais (KERN, 2018, p.122)

Para além das discussões sobre a qualidade das informações na Wikipédia, buscaremos demonstrar a relevância desse sistema sociotécnico para o campo da ciência da informação, focando sobretudo no tema dos museus brasileiros, já que a Wikipédia é uma rica fonte de informações acerca das interações humanas na construção e curadoria coletiva de conhecimentos sobre o mundo. Uma vez que as relações são mediadas por sistemas que registram dados sobre as interações, a Wikipédia se revela uma fonte promissora para a observação de fenômenos sociais relacionados às interações coletivas e colaborativas na busca pela construção e socialização do saber.

\section{Wikipédia e a produção colaborativa de conteúdo}

A Wikipédia é uma das iniciativas precursoras das novas práticas de produção de informação e conhecimento emergentes no início do século XXI, a partir do desenvolvimento da chamada web 2.0 ou web social. O surgimento da web 2.0 ou web social é marcado pelo estabelecimento da web enquanto plataforma ${ }^{6}$ e pelo surgimento de serviços que tornam possíveis a interação do usuário e a produção colaborativa de conteúdos, o que não era possível nos serviços da web 1.o.

A web 1.0 era estruturada por sites que apresentavam o conteúdo de forma estática, sem oferecer possibilidades de interação do usuário com os conteúdos. Com o

\footnotetext{
${ }^{6}$ Serviços online.
} 
surgimento de serviços baseados na web 2.0, os ambientes começaram a se apresentar de forma mais aberta e dinâmica, permitindo a participação e colaboração dos usuários na produção dos conteúdos, e como resultado possibilitou a criação da conexão de comunidades de usuários com interesses em comum (BLATTMANN; SILVA, 2007, p. 199).

Nesse contexto, a Wikipédia surge como uma plataforma de produção de conteúdo coletivo e colaborativo que, segundo D'Ándrea, adota "um modelo aberto e horizontalizado de produção, claramente influenciado pelo paradigma do software livre e pela cultura hacker" (2009, p.76), podendo ser classificada como um site User Generate Content ${ }^{7}$ (UCG).

Ao contrário de outros sites de UGC, como YouTube (compartilhamento de vídeos) e Flickr (fotos), em que o material publicado por cada usuário não interfere diretamente nas escolhas e nas preferências dos demais, o gerenciamento da Wikipédia é especialmente complexo devido à necessidade de chegar a um consenso sobre o melhor formato e conteúdo para cada artigo (D'ANDREA, 2009, p.76).

O modelo de produção de conteúdo proposto pela Wikipédia é baseado na produção e curadoria coletiva e colaborativa. O slogan "A enciclopédia livre que todos podem editar" (WIKIMEDIA FOUNDATION, 2018) convida os usuários a fazerem suas contribuições, incentivando-os a compartilhar seus conhecimentos de forma sistematizada e orientada. A contribuição pode ser realizada sem necessidade de cadastro do usuário no sistema, mas para que ela seja mantida é necessário que um conjunto de princípios ${ }^{8}$ e boas práticas sejam observados. Esse conjunto de princípios e práticas é estabelecido por políticas da Wikipédia e pelos processos de tomada de decisão da comunidade. Cada versão da Wikipédia possui regras próprias de publicação de acordo com as decisões da comunidade linguística envolvida em sua produção.

Os processos de verificação e validação das informações na Wikipédia são realizados por sua comunidade de colaboradores, os wikipedistas e por algoritmos programados por editores humanos, os bots. Segundo Gonçalves (2014), os indivíduos que compõem essas comunidades, os wikipedistas, podem ser vistos como "atores que formam redes sociais mediadas pela estrutura de recursos incitados pela Wikipédia" (2014, p.13). Dessa forma, a rede social composta pelos wikipedistas se articula e se organiza em torno do interesse dos atores em compartilhar informações, produzir conteúdos e garantir a qualidade das informações, realizando revisões, executando edições e se engajando nos fóruns de discussões.

A comunidade ou as comunidades ${ }^{9}$ de colaboradores que realizam esses processos são formadas por diferentes níveis hierárquicos, determinados pela quantidade e tipo de contribuição, o que configura uma rígida estrutura organizacional (D'ANDREA, 2009, p.77).

A produção coletiva de conhecimento e as interações entre atores nesse sistema sociotécnico é passível de ser verificada e quantificada por meio da análise dos

\footnotetext{
${ }^{7}$ Conteúdo gerado por usuário.

${ }^{8}$ Balizados por cinco pilares: o enciclopedismo, a neutralidade de ponto de vista, a licença livre, a boa convivência e a liberalidade nas regras.

${ }^{9}$ Uma vez que cada Wikipédia possui uma comunidade específica devido à separação em idiomas.
} 
registros das atividades na plataforma e de ferramentas criadas para tal fim. Dessa forma, a Wikipédia configura-se como uma importante fonte de pesquisa e pode nos revelar algumas informações acerca das dinâmicas da produção coletiva de conhecimento, compartilhamento e curadoria de informações em rede.

\section{A presença dos museus brasileiros na Wikipédia}

Em um contexto no qual a internet se torna um dos principais meios de troca e exposição de informações, a Wikipédia se apresenta como um meio digital estratégico para as instituições culturais, sobretudo para os museus. Como a Wikipédia constitui-se atualmente como a principal fonte de informações enciclopédicas para os usuários que navegam na web em busca de informação, a "enciclopédia livre" torna-se um recurso potente de difusão de informações sobre os museus na internet para um público amplo.

Ao consultar a Wikipédia em português, e buscar a categoria" “museus", é possível ter acesso ao link "Lista de museus", que apresenta uma coletânea de museus que contém informações presentes na Wikipédia. Dentro dessa lista, é possível ter acesso à "Lista de museus do Brasil" "11 e visualizar os museus por estado, como se pode observar na imagem abaixo.

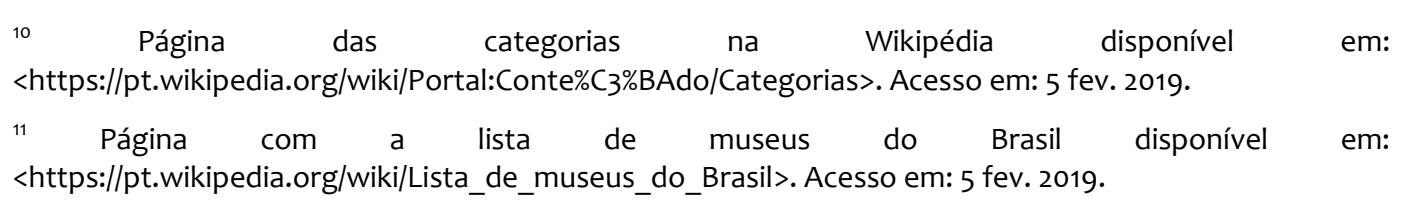


Figura 1 - Página da Lista de museus do Brasil na Wikipédia.
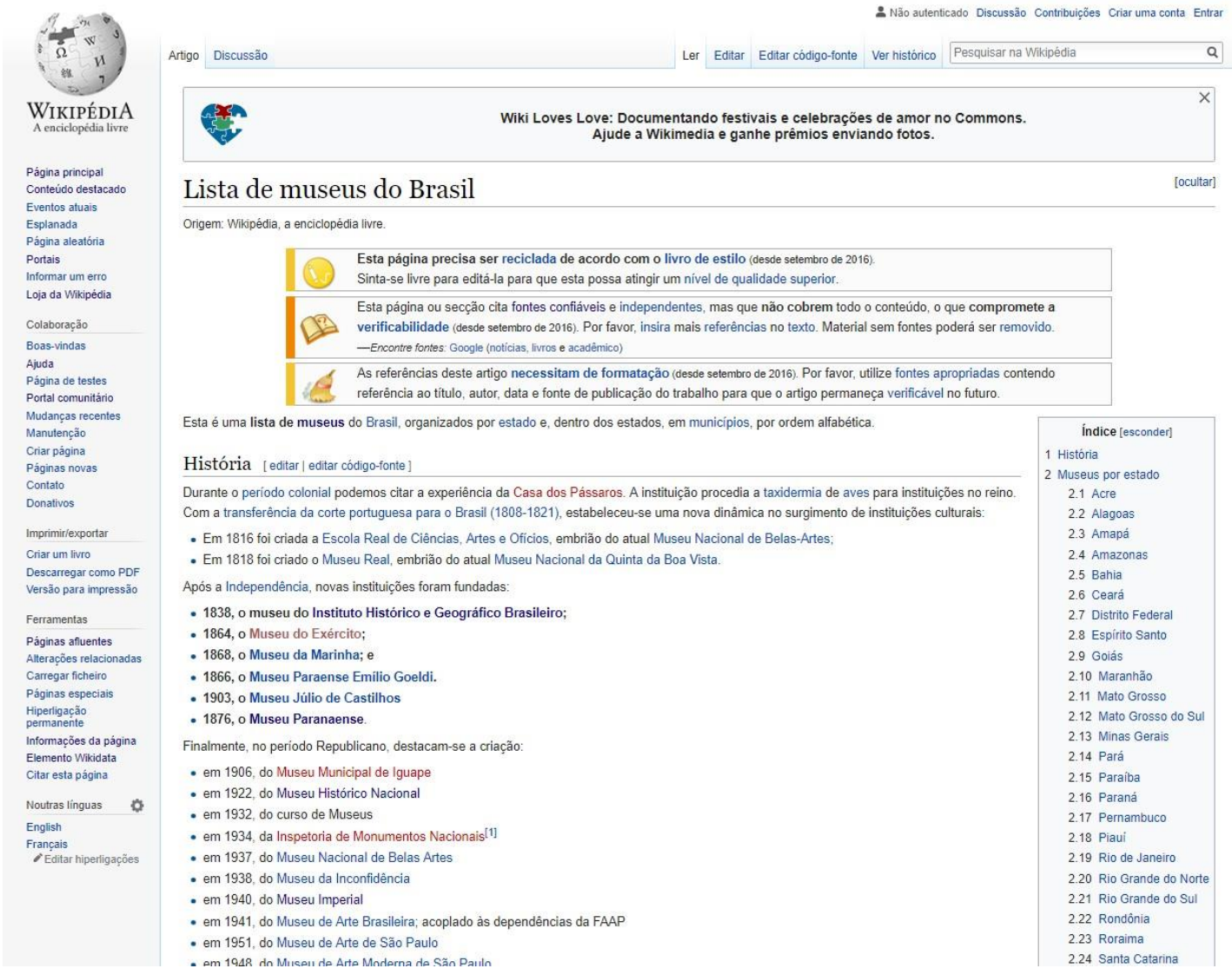

Lista de museus do Brasil

origem: Wikipédia, a enciclopédia livre

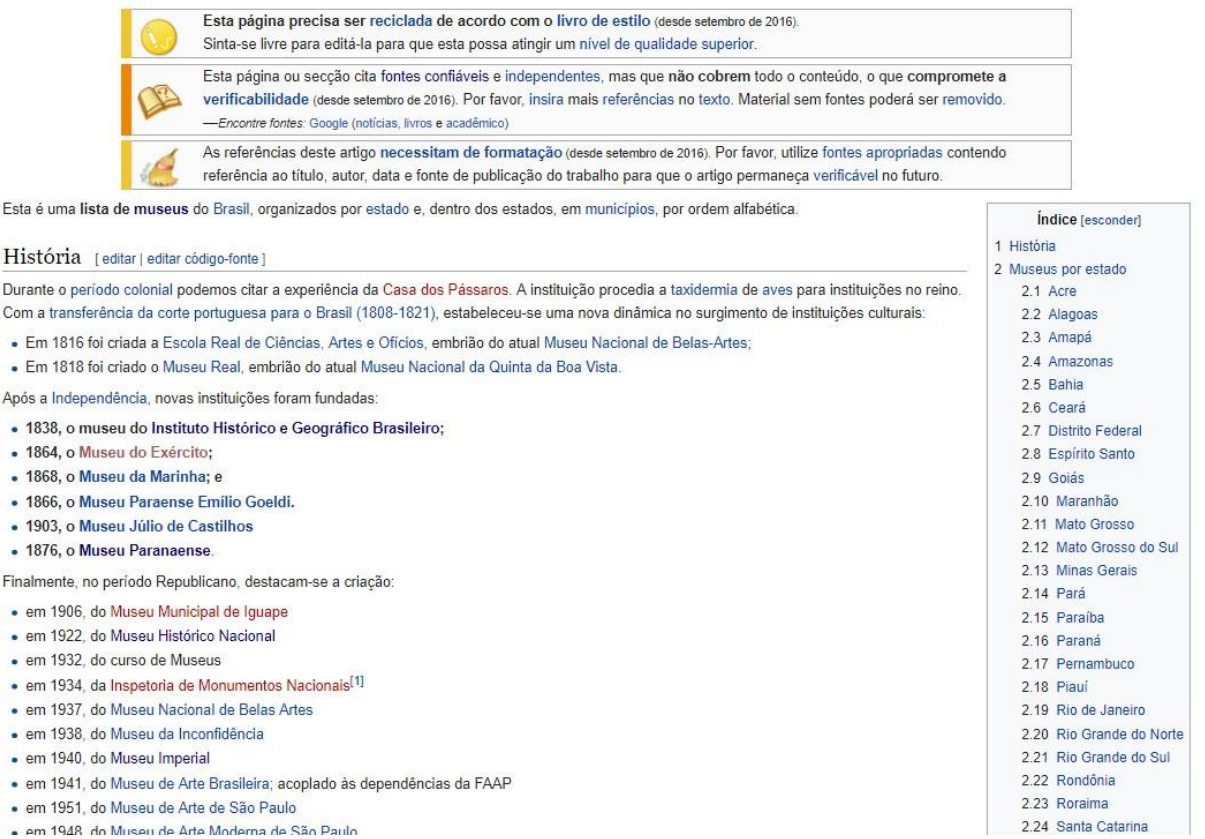

Fonte: Wikipédia (2019).

Para ter uma visão geral da presença de artigos sobre os museus na Wikipédia, coletamos os dados dessa lista, colocamos em uma planilha e realizamos a limpeza dos links em vermelho (que indicam que o nome do museu foi citado, porém não possui um artigo na Wikipédia). Com base nos dados obtidos por meio da "Lista de museus do Brasil", foi possível identificar que a Wikipédia em português apresenta, pelo menos, 457 artigos $^{12}$ sobre museus brasileiros.

É importante ressaltar que o resultado obtido por meio dessa lista é apenas uma amostra. Uma vez que os dados apresentados na lista não são automáticos, sendo uma coletânea construída pelos usuários, pode não representar a totalidade de artigos sobre os museus presentes na Wikipédia. Para a obtenção do total de artigos sobre os museus brasileiros seria necessário uma pesquisa mais detalhada e aprofundada sobre esse problema específico. Entretanto, por meio desta amostra é possível observar que a comunidade de colaboradores do projeto da Wikipédia em português têm empreendido esforços no sentido de produzir informações sobre as instituições museológicas brasileiras.

\footnotetext{
${ }^{12}$ Os dados extraídos da Lista de Museus na Wikipédia referente aos artigos sobre museus em edição, estão disponíveis para consulta: <https://docs.google.com/spreadsheets/d/1G7ELykq6XplavKJladgxoVGD1DKCBojZQEVDuy7k5Al/edit?usp =sharing $>$.
} 
O processo de participação na edição dos artigos do museus pode se mostrar assimétrico, sendo que algumas páginas possuem muitas informações sobre a instituição e outras menos. Como exemplo dessas desigualdades estruturais podemos citar o artigo sobre o Museu Histórico Nacional e o artigo do Museu de Arte Sacra da Boa Morte.

O artigo sobre o Museu Histórico Nacional mostra-se de forma mais estruturada, sendo composto de uma breve apresentação do museu, sua história e características, informações sobre o seu acervo, visitações e exposições, uma lista de diretores, indicações de bibliografia sobre o museu, e ligações externas que direcionam para o site da instituição e para anais em sua biblioteca virtual, além das referências bibliográficas, item obrigatório nos artigos da Wikipédia. Já o artigo sobre o Museu de Arte Sacra da Boa Morte se apresenta de forma menos estruturada, apresenta uma breve apresentação do museu e as referências bibliográficas.

Figura 2 - Página do artigo sobre o Museu Histórico Nacional na Wikipédia.

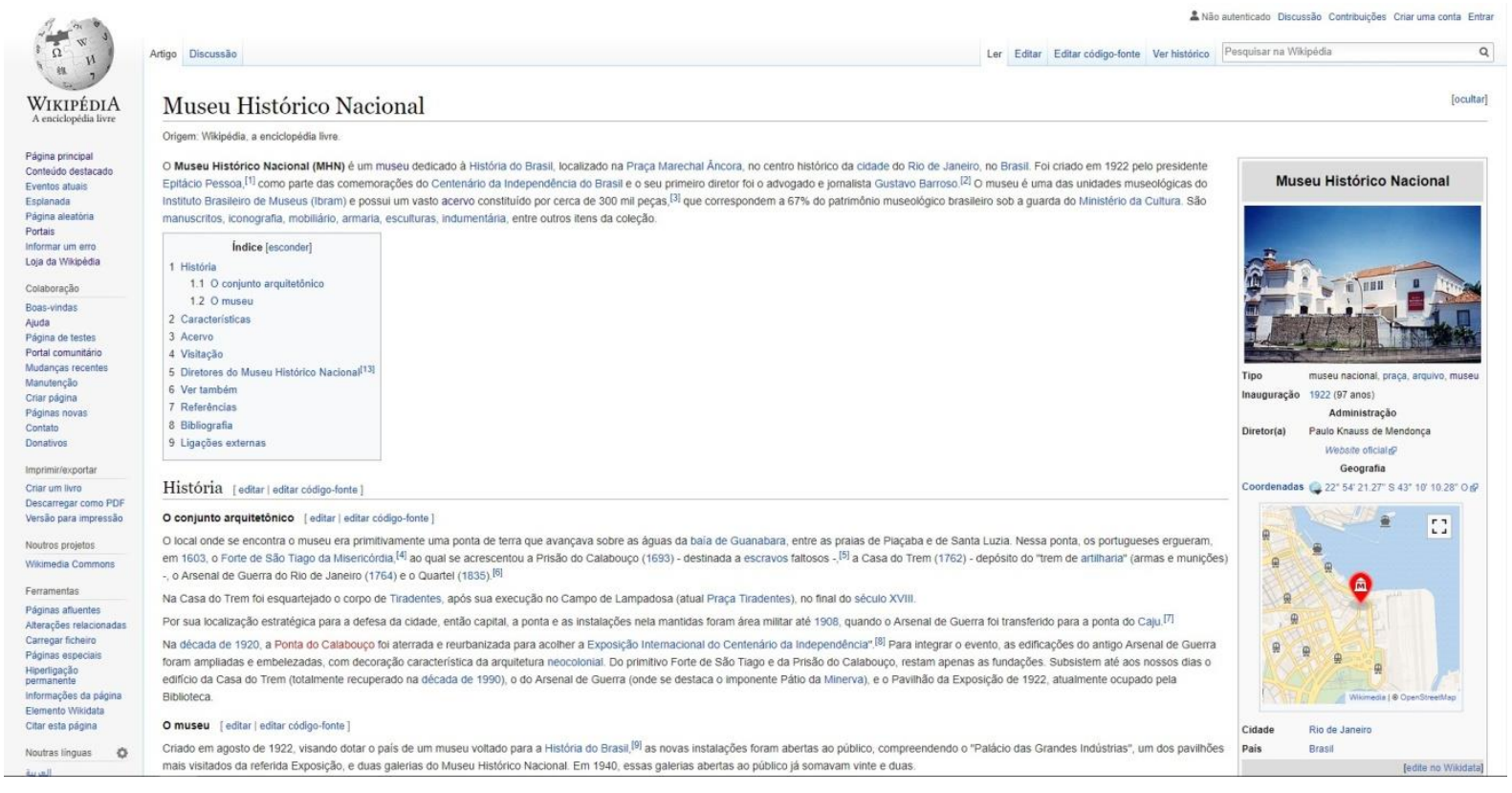

Fonte: Wikipédia (2019). 
Figura 3 -Página do artigo sobre o Museu de Arte Sacra da Boa Morte na Wikipédia.

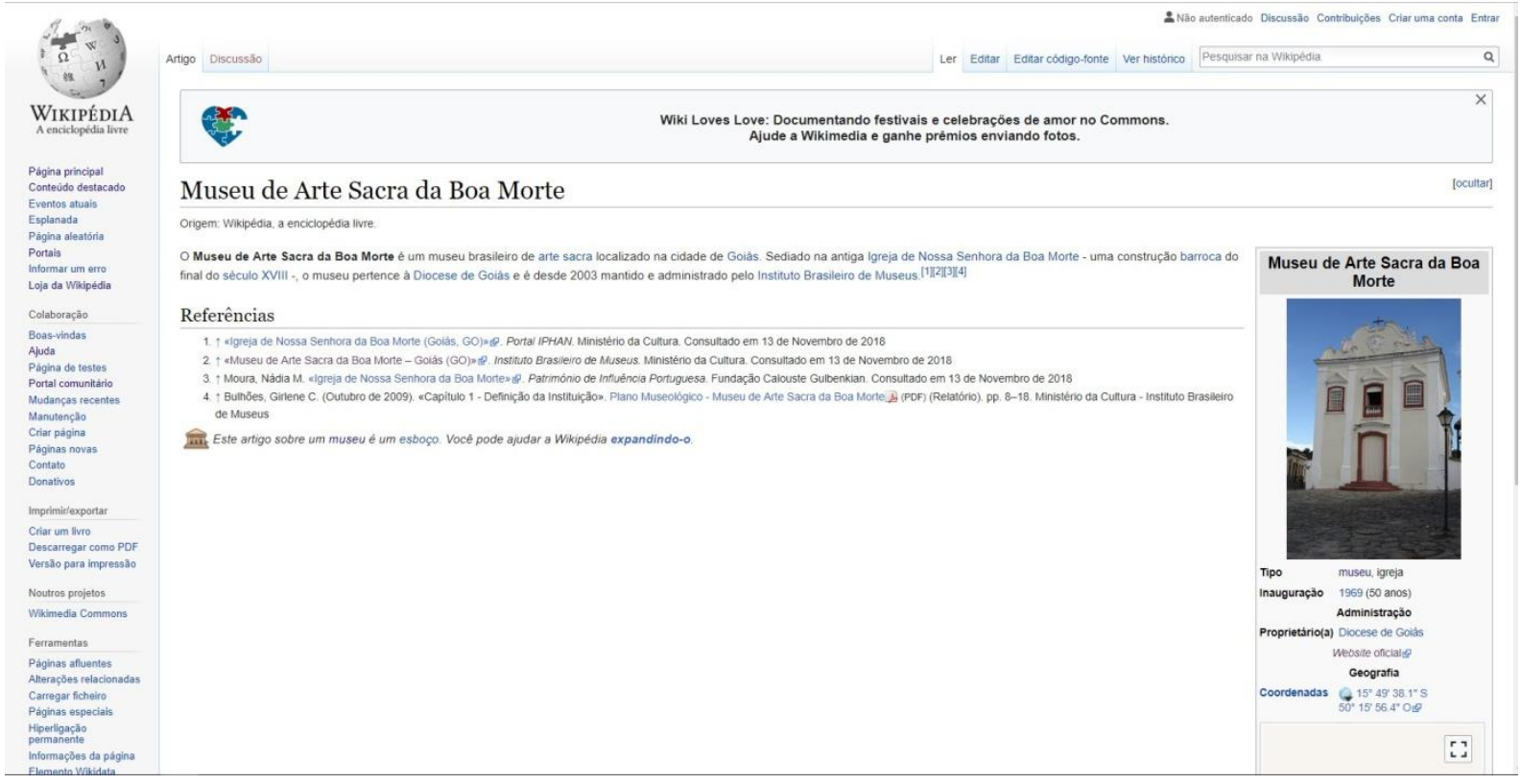

Fonte: Wikipédia (2019).

Nesse contexto, a presente pesquisa tem como objetivo identificar e descrever alguns aspectos da dinâmica de produção colaborativa e coletiva de informação sobre os museus brasileiros na Wikipédia. É possível compreender aqui que a Wikipédia se constitui uma fonte de informação construída socialmente e que demonstra uma das facetas da presença digital dos museus em rede em um espaço de sociabilidade do qual pouco ainda se conhece em relação aos museus brasileiros. Dessa maneira, o objetivo da presente pesquisa visa descrever como se dá a dinâmica social de produção dessa fonte de informação e refletir como o próprio campo museal poderia se apropriar desse espaço como mais um ambiente para o exercício de sua função social. Para isso, utilizaremos como amostra para essa análise os 29 museus brasileiros sob administração direta do Instituto Brasileiro de Museus (Ibram).

\section{METODOLOGIA}

O presente artigo tem como objetivo apresentar uma descrição acerca da dinâmica de produção coletiva de informações sobre os museus brasileiros vinculados ao Ibram na Wikipédia, refletindo sobre seus possíveis efeitos para o campo museal. Para isso, foi realizada a coleta de dados quantitativos sobre determinados aspectos da produção dos artigos sobre esses museus. Os dados coletados foram os seguintes: a) quais e quantos museus sob administração direta do Ibram possuem artigos sobre eles na Wikipédia; b) os links dos artigos dos museus na Wikipédia; c) concentração artigos de museus do Ibram por unidade federativa; d) total de edições nas páginas; e) número de usuários envolvidos nas edições; f) datas da primeira e última edição; g) número de edições por ano; h) número de links que referenciam o artigo em outros artigos da Wikipédia e número de links que o artigo referencia; e i) o nome do usuário dos 10 editores que mais participaram da edição de cada artigo, visando identificar os mais atuantes. A coleta de dados foi realizada entre os dias 28 de maio e 7 de junho de 2018. 
Para a coleta de links dos artigos sobre museus presentes na Wikipédia, foi realizada a consulta ao artigo sobre o lbram $^{13}$ na Wikipédia, no qual foi possível ter acesso à relação de links do artigo de cada um dos museus. Com os links e a relação dos nomes dos artigos sobre os museus, foi possível coletar os dados estatísticos das páginas utilizando o software XTools.

O software XTools é um conjunto de ferramentas estatísticas para Wikis desenvolvida pela Fundação Wikimedia. ${ }^{14}$ Por meio da instalação do XTools da Wikipédia, ${ }^{15}$ é possível se obterem diversos dados numéricos dos artigos da Wikipédia e das ações dos editores. Para isso, o XTools disponibiliza as seguintes ferramentas: a) Contador de Edição, que permite a análise das contribuições do usuário; b) Histórico da Páginas, que apresenta várias estatísticas referentes ao histórico da página desejada; c) Principais Edições, que apresenta dados de edições por usuários; d) Edições Automáticas, apresenta dados sobre edições automatizadas e não automatizadas; e) Edição de Categoria, apresenta os dados de edição de uma categoria específica; f) Estatísticas de Administração, mostra dados referentes às ações dos administradores; g) Edição de Sumário, mostra dados de uso da edição de sumários; h) Contador Simples, apresenta um contador simples de edições.

Para a coleta de dados do presente trabalho, utilizamos a ferramenta "Histórico de Páginas". A ferramenta de busca "Histórico de Páginas" possui quatro campos para inserção de parâmetros. Utilizamos para a coleta os dois primeiros: "Projeto" e "Título da Página". No campo "Projeto", deve ser informado qual o projeto da Wikipédia que se deseja coletar os dados. No presente caso, definiu-se o projeto pt.wikipedia.org, que é o domínio brasileiro das páginas dos museus. No campo "Título da Página", foi inserido (em consultas individuais) os títulos das páginas selecionadas para a coleta de dados, no caso o nome dos museus do Ibram. $\mathrm{Na}$ ferramenta, é possível se delimitar a busca por determinado período de tempo. Entretanto, esse recurso não foi utilizado em nossa coleta, pois a intenção foi obter desde os primeiros dados registrados até os últimos disponíveis.

De acordo com o site do software XTools, é possível se coletarem os dados automaticamente por meio de uma API, ${ }^{16}$ porém não foi utilizada como meio neste trabalho devido à quantidade reduzida de museus que compõem a pesquisa, mas é o caminho mais adequado para pesquisas que queiram coletar informações de um grande número de artigos, e que se pretende explorar em trabalhos futuros.

Os dados coletados por meio do XTools foram tabulados e organizados ${ }^{17}$ nas Planilhas Google, um serviço online baseado em nuvem. Dessa forma, foram utilizadas três tabelas em abas distintas. Na primeira aba, foi construída uma tabela chamada "Info dos Museus", contendo informações e dados gerais dos artigos, tais como: os links do XTools com os dados numéricos consultados; total de edições

13 Artigo sobre o lbram na Wikipédia disponível em: <https://pt.wikipedia.org/wiki/Instituto_Brasileiro_de_Museus>. Acesso em: 5 jan. 2019.

${ }^{14}$ Mais informações sobre o software Xtools disponíveis em: <https://xtools.wmflabs.org/articleinfo>. Acesso em: 5 jan. 2019.

${ }^{15}$ Disponível em:< https://xtools.wmflabs.org/>. Acesso em: 5 jan. 2019.

16 Informações sobre a API da XTools disponíveis em: <https://xtools.readthedocs.io/en/stable/api/index.htm>. Acesso em: 5 jan. 2019.

17 Os dados da pesquisa estão disponíveis para consulta em: <https://docs.google.com/spreadsheets/d/1tjl7LQCcscDPQISm_GrBEKLEuWAIWHDcoV52R7OsN8/edit?us $\mathrm{p}=$ sharing $>$. Acesso em: 5 jan. 2019. 
realizadas; quantidade de editores; datas da primeira e última edição; números de links que saem dessa página para outros artigos da Wikipédia; links de outros artigos da Wikipédia que levam para esse artigo. Na tabela chamada "Top Editores", foram inseridos dados como o nome de usuário dos 10 maiores editores da página, os links das páginas desses usuários e as datas de edição. Na terceira tabela, chamada "Contagem de Edições por Ano", foram distribuídos os nomes dos museus junto à frequência de edições nessas páginas por ano.

Vale dizer que para melhorar a compreensão da dinâmica social de construção das páginas, realizou-se uma análise de rede por meio do software Gephi ${ }^{18}$ para verificar como os "Top Editores" participaram da edição de mais de uma página de museu. Com essa análise, é possível perceber a influência desses editores e analisar se há aqui a formação de uma eventual comunidade virtual de editores que se repetem nesse trabalho de curadoria das informações museais na Wikipédia. Os dados coletados e tabulados foram posteriormente submetidos à análise. Na próxima seção, as análises serão apresentadas e os resultados descritos.

\section{RESULTADOS}

A partir da coleta, tabulação e análise dos dados, foi possível observar que dos 29 museus listados na página do Ibram, 9 não possuem um artigo sobre eles na Wikipédia (quadro abaixo). Dos museus com artigos na Wikipédia, 10 estão localizados no estado do Rio de Janeiro e 4 em Minas Gerais. Os demais estados Espírito Santo, Goiás, Pernambuco, Rio Grande do Sul, Santa Catarina e São Paulo possuem apenas um museu com verbete.

Quadro 1- Informações gerais sobre os museus do Ibram e links na Wikipédia.

\begin{tabular}{|c|c|c|c|c|c|}
\hline$\#$ & Museu & Inauguração & Município & Estado & Link na Wikipédia \\
\hline 1 & Palácio Rio Negro & 2010 & Petrópolis & RJ & $\begin{array}{l}\text { https://pt.wikipedia.org/wiki/Pal\%C3\% } \\
\text { A1cio_Rio_Negro }\end{array}$ \\
\hline 2 & Museu Villa-Lobos & 1960 & Rio de Janeiro & RJ & Não possui \\
\hline 3 & Museu Victor Meirelles & 1952 & Florianópolis & SC & $\begin{array}{l}\text { https://pt.wikipedia.org/wiki/Museu_ } \\
\text { Victor_Meirelles }\end{array}$ \\
\hline 4 & Museu Solar Monjardim & 1980 & Vitória & ES & $\begin{array}{l}\text { https://pt.wikipedia.org/wiki/Museu_ } \\
\text { Solar_Monjardim }\end{array}$ \\
\hline 5 & $\begin{array}{l}\text { Museu Socioambiental de } \\
\text { Itaipu }\end{array}$ & 1977 & Niterói & RJ & $\begin{array}{l}\text { https://pt.wikipedia.org/wiki/Museu_ } \\
\text { de_Arqueologia_de_Itaipu }\end{array}$ \\
\hline 6 & $\begin{array}{l}\text { Museu Regional de São } \\
\text { João del-Rei }\end{array}$ & 1963 & São João del-Rei & MG & $\begin{array}{l}\text { https://pt.wikipedia.org/wiki/Museu_ } \\
\text { Regional_de_S\%C3\%A30_Jo\%C3\%A30_ } \\
\text { del-Rei }\end{array}$ \\
\hline 7 & Museu Regional de Caeté & 1950 & Caeté & MG & Não possui \\
\hline 8 & $\begin{array}{l}\text { Museu Nacional de Belas } \\
\text { Artes }\end{array}$ & 1937 & Rio de Janeiro & RJ & $\begin{array}{l}\text { https://pt.wikipedia.org/wiki/Museu_ } \\
\text { Nacional_de_Belas_Artes_\%28Brasil\% } \\
29\end{array}$ \\
\hline 9 & Museu Lasar Segall & 1967 & São Paulo & $\mathrm{SP}$ & $\begin{array}{l}\text { https://pt.wikipedia.org/wiki/Museu_ } \\
\text { Lasar_Segall }\end{array}$ \\
\hline
\end{tabular}

${ }^{18}$ Informações sobre o Gephi disponíveis em:< https://gephi.org/>. Acesso em: 10 fev. 2019. 


\begin{tabular}{|c|c|c|c|c|c|}
\hline 10 & Museu Imperial & 1943 & Petrópolis & RJ & $\begin{array}{l}\text { https://pt.wikipedia.org/wiki/Museu_ } \\
\text { Imperial }\end{array}$ \\
\hline 11 & Museu Histórico Nacional & 1922 & Rio de Janeiro & RJ & $\begin{array}{l}\text { https://pt.wikipedia.org/wiki/Museu_ } \\
\text { Hist\%C3\%B3rico_Nacional }\end{array}$ \\
\hline 12 & $\begin{array}{l}\text { Museu Forte Defensor } \\
\text { Perpétuo }\end{array}$ & 1957 & Paraty & RJ & Não possui \\
\hline 13 & Museu do Ouro & 1946 & Sabará & MG & $\begin{array}{l}\text { https://pt.wikipedia.org/wiki/Museu_ } \\
\text { do_Ouro_(Sabar\%C3\%A1) }\end{array}$ \\
\hline 14 & Museu do Diamante & 1954 & Diamantina & MG & Não possui \\
\hline 15 & Museu do Açude & 1963 & Rio de Janeiro & RJ & $\begin{array}{l}\text { https://pt.wikipedia.org/wiki/Museu_ } \\
\text { do_A\%C3\%A7ude }\end{array}$ \\
\hline 16 & $\begin{array}{l}\text { Museu de Arte Sacra de } \\
\text { Paraty }\end{array}$ & 1978 & Paraty & RJ & Não possui \\
\hline 17 & $\begin{array}{l}\text { Museu de Arte Sacra da } \\
\text { Igreja da Boa Morte }\end{array}$ & 1969 & Goiás & GO & $\begin{array}{l}\text { https://pt.wikipedia.org/wiki/Museu_ } \\
\text { de_Arte_Sacra_da_Boa_Morte }\end{array}$ \\
\hline 18 & \begin{tabular}{|l|} 
Museu de Arte Religiosa e \\
Tradicional de Cabo Frio
\end{tabular} & 1982 & Cabo Frio & RJ & Não possui \\
\hline 19 & Museu das Missões & 1940 & $\begin{array}{l}\text { São Miguel das } \\
\text { Missões }\end{array}$ & RS & $\begin{array}{l}\text { https://pt.wikipedia.org/wiki/Museu_ } \\
\text { das_Miss\%C3\%B5es }\end{array}$ \\
\hline 20 & Museu das Bandeiras & 1949 & Goiás & GO & $\begin{array}{l}\text { https://pt.wikipedia.org/wiki/Museu_ } \\
\text { das_Bandeiras }\end{array}$ \\
\hline 21 & Museu da República & 1960 & Rio de Janeiro & RJ & $\begin{array}{l}\text { https://pt.wikipedia.org/wiki/Pal\%C3\% } \\
\text { A1cio_do_Catete }\end{array}$ \\
\hline 22 & Museu da Inconfidência & 1946 & Ouro Preto & MG & $\begin{array}{l}\text { https://pt.wikipedia.org/wiki/Museu_ } \\
\text { da_Inconfid\%C3\%AAncia }\end{array}$ \\
\hline 23 & Museu da Chácara do Céu & 1972 & Rio de Janeiro & RJ & $\begin{array}{l}\text { https://pt.wikipedia.org/wiki/Museu_ } \\
\text { da_Ch\%C3\%A1cara_do_C\%C3\%Agu }\end{array}$ \\
\hline 24 & Museu da Abolição & 1983 & Recife & PE & $\begin{array}{l}\text { https://pt.wikipedia.org/wiki/Museu_ } \\
\text { da_Aboli\%C3\%A7\%C3\%A30 }\end{array}$ \\
\hline 25 & $\begin{array}{l}\text { Museu Casa Histórica de } \\
\text { Alcântara }\end{array}$ & 2004 & Alcântara & MA & Não possui \\
\hline 26 & $\begin{array}{l}\text { Museu Casa de Benjamin } \\
\text { Constant }\end{array}$ & 1982 & Rio de Janeiro & RJ & $\begin{array}{l}\text { https://pt.wikipedia.org/wiki/Museu_ } \\
\text { Casa_de_Benjamin_Constant }\end{array}$ \\
\hline 27 & Museu Casa da Hera & 1968 & Vassouras & RJ & $\begin{array}{l}\text { https://pt.wikipedia.org/wiki/Museu_ } \\
\text { Casa_da_Hera }\end{array}$ \\
\hline 28 & Casa dos Ottoni & 1949 & Serro & MG & $\begin{array}{l}\text { https://pt.wikipedia.org/wiki/Casa_do } \\
\text { s_Ottoni }\end{array}$ \\
\hline 29 & Casa da Princesa & - & Pilar de Goiás & GO & Não possui \\
\hline
\end{tabular}

Fonte: Elaborado pelos autores (2019).

Em relação à comunidade de editores que colaboraram em artigos dos 20 museus do Ibram na Wikipédia, foi possível identificar a participação de um total de 555 usuários, que realizaram um total de 1.108 edições nas páginas. O Museu Imperial teve o maior número de colaboradores (167), seguido pelo Museu Histórico Nacional (148) e pelo museu Museu Lasar Segall (111), como pode ser verificado pelo quadro abaixo. 
Quadro 2 - Total de edições, editores e datas da primeira e última edição.

\begin{tabular}{|c|c|c|c|c|c|c|c|}
\hline$\#$ & Museu & $\begin{array}{l}\text { Total de } \\
\text { Edições }\end{array}$ & $\mid \begin{array}{lr}\mathrm{N}^{\circ} & \mathrm{de} \\
\text { Editores }\end{array}$ & $\begin{array}{l}\text { Média de } \\
\text { Edições por } \\
\text { Editores }\end{array}$ & $\begin{array}{l}\text { Primeira } \\
\text { Edição }\end{array}$ & $\begin{array}{l}\text { Última } \\
\text { Edição }\end{array}$ & $\begin{array}{l}\text { Anos de } \\
\text { Edição }\end{array}$ \\
\hline 1 & Museu Imperial & 167 & 100 & 1,6 & 2007-07-08 & $2018-04-25$ & 11 \\
\hline 2 & $\begin{array}{ll}\text { Museu } & \text { Histórico } \\
\text { Nacional } & \end{array}$ & 149 & 66 & 2,2 & 2004-07-06 & 2018-03-14 & 14 \\
\hline 3 & Museu Lasar Segall & 111 & 71 & 1,1 & $2004-07-08$ & $2018-04-28$ & 14 \\
\hline 4 & Museu da Inconfidência & 103 & 55 & 1,8 & $2005-10-30$ & $2009-08-10$ & 4 \\
\hline 5 & Museu do Açude & 83 & 25 & 3,3 & $2007-10-05$ & 2017-09-2 & 10 \\
\hline 6 & Museu da Abolição & 71 & 26 & 2,7 & $2007-07-25$ & $2018-04-26$ & 11 \\
\hline 7 & Palácio Rio Negro & 71 & 45 & 1,5 & $2005-08-28$ & 2007-07-11 & 2 \\
\hline 8 & $\begin{array}{l}\text { Museu Socioambiental } \\
\text { de Itaipu }\end{array}$ & 55 & 24 & 2,1 & 2005-10-09 & 2017-04-14 & 12 \\
\hline 9 & Museu Solar Monjardim & 55 & 11 & 2,2 & 2009-09-13 & $2018-05-03$ & 9 \\
\hline 10 & $\begin{array}{l}\text { Museu da Chácara do } \\
\text { Céu }\end{array}$ & 45 & 15 & 3 & 2008-06-11 & 2017-04-11 & 9 \\
\hline 11 & Museu das Missões & 41 & 18 & 2,2 & $2006-05-08$ & $2018-04-28$ & 12 \\
\hline 12 & Museu Casa da Hera & 40 & 20 & 2 & 2008-02-29 & 2012-10-04 & 4 \\
\hline 13 & Museu Victor Meirelles & 31 & 21 & 1,4 & 2005-09-22 & 2018-01-03 & 3 \\
\hline 14 & Museu da República & 22 & 16 & 1,3 & 2004-07-09 & $2007-02-08$ & 3 \\
\hline 15 & $\begin{array}{l}\text { Museu Regional de São } \\
\text { João del-Rei }\end{array}$ & 18 & 7 & 2,5 & 2010-12-19 & 2017-10-11 & 7 \\
\hline 16 & Museu Casa dos Ottoni & 18 & 12 & 1,5 & 2006-09-22 & 2018-03-18 & 12 \\
\hline 17 & $\begin{array}{l}\text { Museu Casa de Benjamin } \\
\text { Constant }\end{array}$ & 9 & 7 & 1,2 & $2013-02-25$ & 2017-08-02 & 4 \\
\hline 18 & $\begin{array}{l}\text { Museu Nacional de Belas } \\
\text { Artes }\end{array}$ & 9 & 8 & 1,1 & $2008-07-17$ & $2008-07-17$ & 0 \\
\hline 19 & Museu do Ouro & 7 & 5 & 1,4 & $2017-04-28$ & 2017-09-11 & 0,4 \\
\hline 20 & Museu das Bandeiras & 3 & 3 & 1 & 2013-08-19 & 2017-12-13 & 4 \\
\hline
\end{tabular}

Fonte: elaborado pelos autores (2019).

Essa tabela apresenta dados relativos à interação entre a comunidade de editores e os artigos dos museus. De início, percebe-se uma grande desigualdade em termos de edições realizadas e editores participantes entre os museus, demonstrando uma diferença significativa em termos de apropriação social do espaço de construção das informações a respeito dos museus. No entanto, quando se observa a média de edições por usuário, percebe-se que esse número se aproxima bastante em relação à amostra, ou seja, a média geral é 1,7 edição por participante, mostrando que na maioria dos casos os participantes realizam em torno de duas edições em cada página, sendo as páginas mais completas aquelas que atraíram mais participantes. Há uma conclusão necessária a se fazer sobre esse resultado, demonstrando que é a dinâmica de participação social que gera uma maior riqueza descritiva em cada página, ampliando detalhes e complementaridades entre os editores de cada verbete. 
Em relação às primeiras edições realizadas nos artigos, a edição mais antiga data de 6 de julho de 2004, no artigo do Museu Histórico Nacional, e a mais recente (levando em consideração o período da coleta dos dados) data de 28 de abril de 2017, no artigo do Museu do Ouro. Entre os anos 2004 a 2008, concentram-se o maior número de primeiras edições (14 primeiras edições), apresentando o auge (4 primeiras edições) no ano de 2005. Essas datas coincidem com um período de intensa movimentação do campo museal brasileiros em busca da estruturação de políticas públicas com o estabelecimento da Política Nacional de Museus em 2003, a criação do Departamento de Museus dentro da estrutura do Iphan e a realização da primeira edição do Fórum Nacional de Museus, ambos no ano de 2004. Essa movimentação culmina na criação do Ibram em 2009. Desse modo, podemos inferir que o aquecimento do debate político sobre o campo pode ter refletido na dinâmica de participação social que levou ao processo de edições de artigos sobre os museus brasileiros na Wikipédia, causas essas que demandam estudos futuros a partir de métodos qualitativos para validação.

Pode-se observar, na última coluna, a quantidade de anos que os artigos receberam contribuições. Os artigos do Museu Histórico Nacional e do Museu Lasar Segall recebem contribuições há 14 anos, seguido do Museu Socioambiental de Itaipu, Museu das Missões e o Museu Casa dos Ottoni. Quanto às últimas edições realizadas, a mais antiga data 25 de julho de 2007 e a mais recente data de 3 de maio de 2018. É possível observar uma concentração de últimas edições entre os anos de 2017 e 2018, demonstrando uma rede em plena atividade.

\section{Gráfico 1 - Número de edições por ano.}

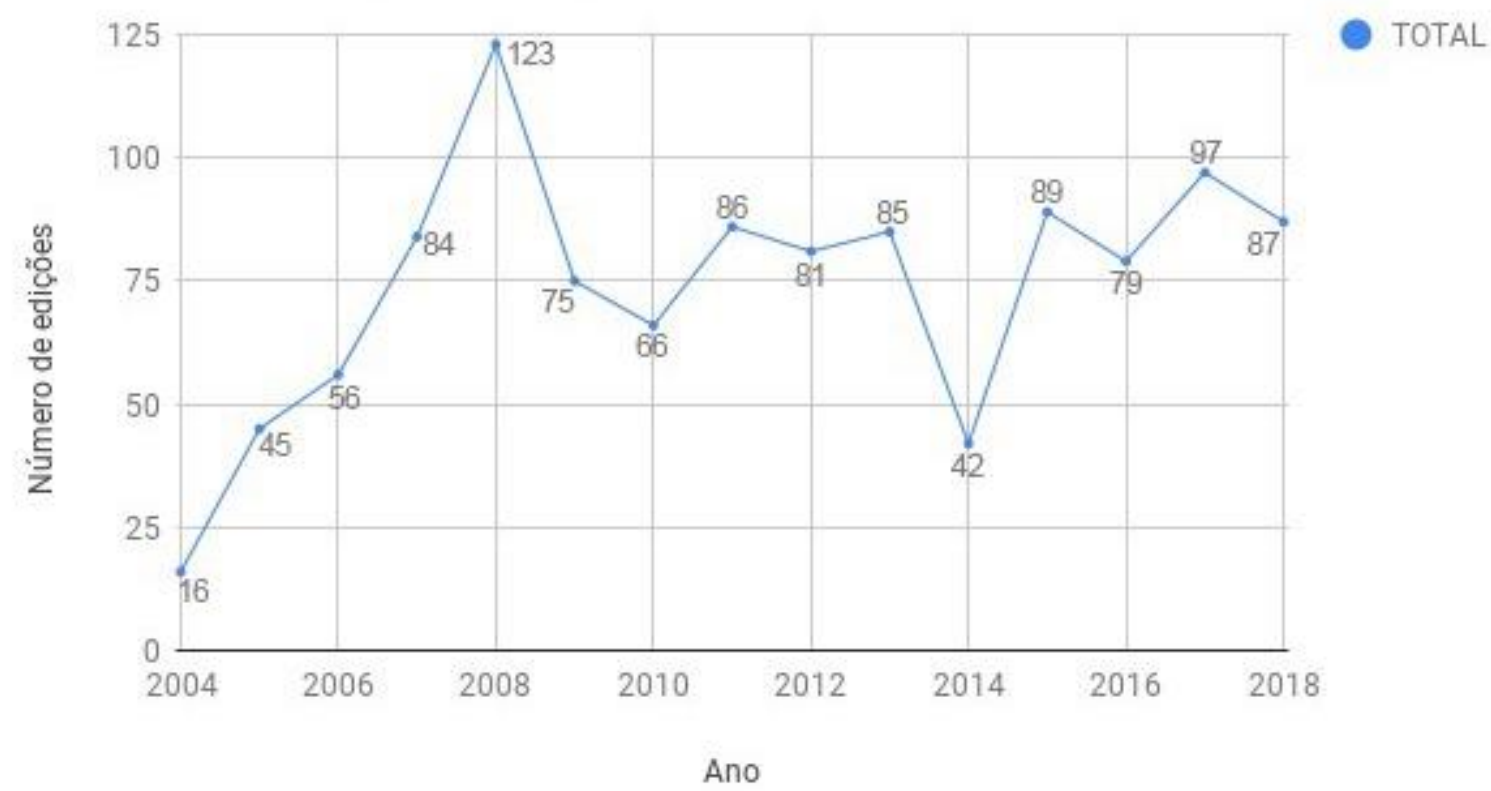

Fonte: elaborado pelos autores (2019).

No gráfico acima, é possível observar a quantidade de edições no total de artigos de museus do Ibram do dia $1^{\circ}$ de janeiro de 2004 até o dia 7 de junho de 2018. O maior número de edições se concentra no ano de 2008 (123), seguido dos anos 2017 (97) e 2015 (89). É possível observar que no ano de 2014 houve a maior baixa no número de edições desde seu apogeu em 2008. Novamente, é interessante observar a intensidade de edições em data próxima à criação do Ibram, talvez denotando o 
aquecimento do debate político sobre a museologia no Brasil e uma reverberação dessa dinâmica em termos de maior participação social sobre o campo na Wikipédia.

Para identificar se os usuários editores chegam a formar uma comunidade de editores, foi verificado se os 10 editores mais ativos em cada artigo ("Top Editores") repetem sua participação em outras páginas. Dessa forma, constatou-se que 18 usuários editaram mais de um dos artigos dos museus do lbram, como demonstra o quadro abaixo.

Quadro 3 - Editores com maior número de participação em diferentes artigos sobre museus.

\begin{tabular}{|l|l|}
\hline Usuário & Páginas de museus editadas \\
\hline Dornicke & 10 \\
\hline Tetraktys & 9 \\
\hline Nice poa & 8 \\
\hline Carlos Luis M C da Cruz & 5 \\
\hline Luizdl & 4 \\
\hline Erico Tachizawa & 3 \\
\hline Hermógenes Teixeira Pinto & 3 \\
\hline Filho & 3 \\
\hline Joalpe & 2 \\
\hline Rjclaudio & 3 \\
\hline Bruno N. Campos & 2 \\
\hline Dbastro & 2 \\
\hline Felipe P & 2 \\
\hline JMGM & 2 \\
\hline Joãofcf & 2 \\
\hline Pedrocolares & 2 \\
\hline Ródi & 3 \\
\hline Thassyaa & 3 \\
\hline Yanguas & 2 \\
\hline
\end{tabular}

Fonte: elaborado pelos autores (2019).

Dos 555 usuários que realizaram ao menos uma edição, chegou-se a 18 (3,2\%) que atuaram em edições em mais de um museu. Pode-se inferir que esse grupo tenha uma proximidade maior com o tema e, eventualmente, um interesse mais genérico na área do que em um respectivo museu, produzindo ações de curadoria que podem contribuir para a disseminação de práticas informacionais no campo. 
Foi construída uma representação gráfica da rede que ilustra a dinâmica de relacionamento entre os "Top Editores" e os artigos. As arestas indicam as relações entre os artigos de museus e os editores. O tamanho do texto indica a representatividade de cada ator (artigo ou usuário) na rede, na medida que concentram menos ou mais relações. Percebe-se como os 18 editores são responsáveis por tecer a rede que conecta diferentes museus, servindo como elemento de articulação social do espaço informacional representado pela rede. São esses editores que, por editarem mais de uma página, podem facilitar a disseminação de práticas de edição entre as páginas dos diferentes museus, eventualmente compartilhando estratégias entre os demais editores. Compreender que tipos de edições esses 18 editores realizaram nas diferentes páginas pelas quais passaram, e como essas estratégias podem ter se disseminado, consiste em tema a ser pesquisado em trabalhos futuros de investigação dessa rede de curadoria.

\section{FIGURA 1 - Representação gráfica da rede de editores por museus.}

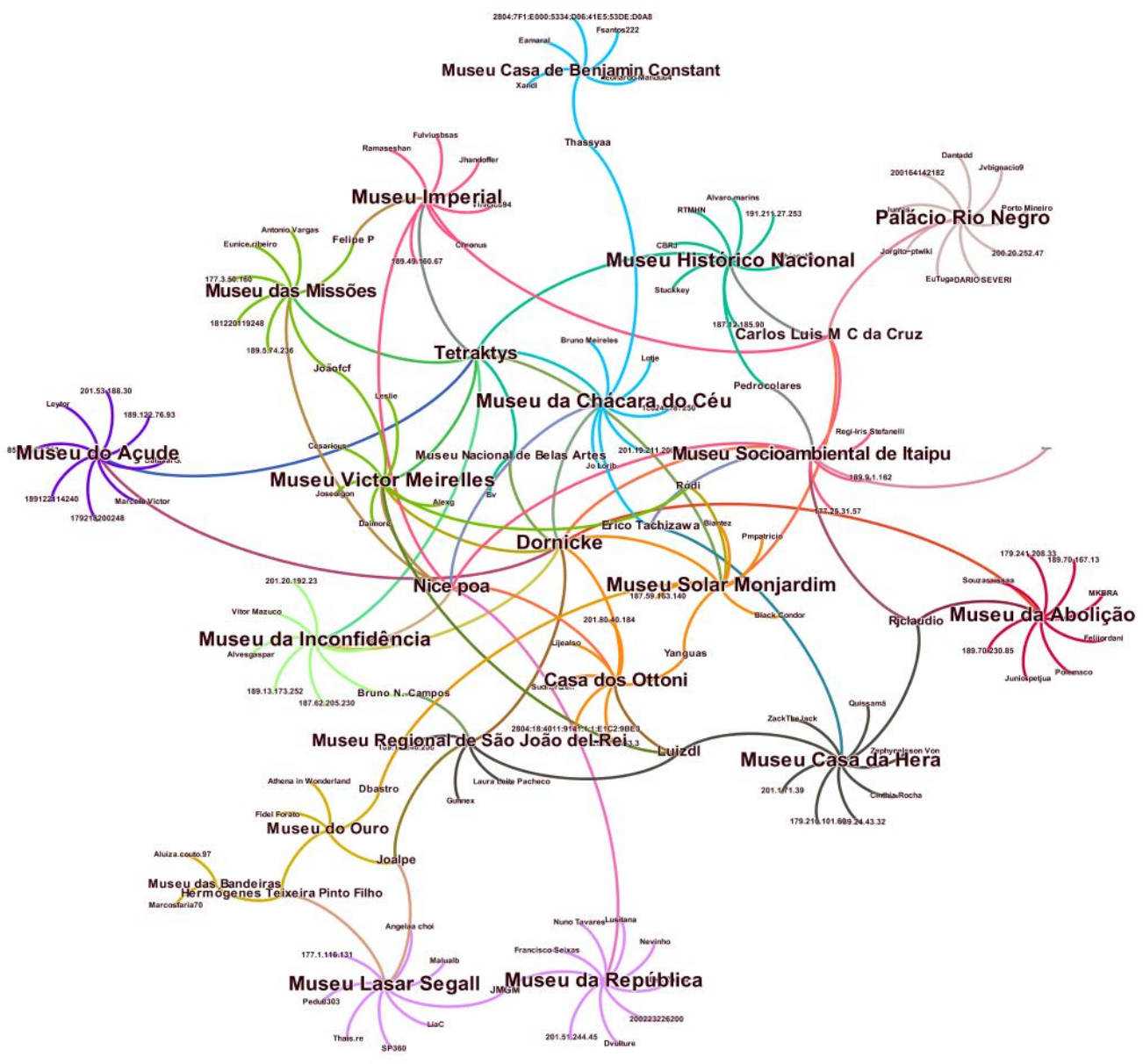

Fonte: elaborado pelos autores (2019).

Por meio da coleta de dados, também foi possível identificar as relações entre os artigos dos museus do Ibram com outros artigos da Wikipédia. No quadro abaixo é possível observar a quantidade de artigos que possuem links que direcionam para os artigos de cada um dos museus, e também a quantidade de links que o artigo do museu apresenta direcionando para outros artigos da Wikipédia. 
Quadro 4 - Número de links que se relacionam ou são relacionados a outros artigos na Wikipédia.

\begin{tabular}{|c|c|c|c|}
\hline Museu & $\begin{array}{l}\text { Links de outros } \\
\text { artigos } \\
\text { (entrada) }\end{array}$ & $\mid \begin{array}{lr}\text { Links } & \text { para } \\
\text { outros } & \text { artigos } \\
\text { (saída) } & \end{array}$ & $\begin{array}{l}\text { Índice } \\
\text { Entrada/Saída }\end{array}$ \\
\hline Museu Lasar Segall & 818 & 411 & 1,9 \\
\hline Museu Histórico Nacional & 211 & 313 & 0,6 \\
\hline Palácio Rio Negro & 107 & 32 & 3,3 \\
\hline Museu Imperial & 107 & 212 & 0,5 \\
\hline Museu Victor Meirelles & 86 & 258 & 0,3 \\
\hline Museu da Inconfidência & 59 & 117 & 0,5 \\
\hline Museu da República & 41 & 1 & 41 \\
\hline Museu Nacional de Belas Artes & 35 & 8 & 4,3 \\
\hline Museu das Missões & 24 & 35 & 0,6 \\
\hline Museu da Chácara do Céu & 23 & 195 & 0,1 \\
\hline Museu da Abolição & 20 & 68 & 0,2 \\
\hline Museu do Açude & 19 & 155 & 0,1 \\
\hline Museu Solar Monjardim & 9 & 32 & 0,2 \\
\hline $\begin{array}{l}\text { Museu Socioambiental de } \\
\text { Itaipu }\end{array}$ & 7 & 57 & 0,1 \\
\hline $\begin{array}{l}\text { Museu Casa de Benjamin } \\
\text { Constant }\end{array}$ & 7 & 12 & 0,5 \\
\hline Museu Casa da Hera & 7 & 62 & 0,1 \\
\hline Casa dos Ottoni & 7 & 27 & 0,2 \\
\hline Museu do Ouro & 6 & 25 & 0,2 \\
\hline $\begin{array}{l}\text { Museu Regional de São João } \\
\text { del-Rei }\end{array}$ & 5 & 7 & 0,7 \\
\hline Museu das Bandeiras & 4 & 26 & 0,1 \\
\hline
\end{tabular}

Fonte: elaborado pelos autores (2019).

Pode-se compreender a dinâmica de links pela ideia de referências que são feitas a um museu (links de outros artigos) e referências que um museu faz em sua página a outros verbetes (links para outros artigos). De início, chama a atenção a desigualdade entre os museus, ressaltando o expressivo número do Museu Lasar Segall como sendo intensamente citado como referência para outros artigos e também citando uma grande quantidade de verbetes. Foge do escopo do presente trabalho 
compreender qualitativamente esse tipo de citação, mas é sem dúvida outro tema que merece trabalhos futuros.

A terceira coluna da tabela apresenta o resultado de divisão entre os links que direcionam para o artigo e os links presentes no artigo que direcionam para outros artigos da Wikipédia. Os resultados acima de 1 indicam que o artigo é mais referenciado por outros artigos do que apresenta referência para outros artigos. Chama a atenção que apenas 4 museus se encontram nessa situação, sendo eles Museu Lasar Segall, Palácio Rio Negro, Museu da República e Museu Nacional de Belas Artes. O resultado tanto pode denotar pouco uso de referências por parte da página do museu em questão para outras página, como o Museu da República (apenas 1 referência), quanto um papel de relevância reconhecida socialmente por muitas citações externas, como o Museu Lasar Segall. Não se percebe aqui uma dinâmica de troca de referências entre páginas, ou seja, nem sempre o museu que cita mais é o que recebe mais citação. Essa é uma dinâmica que merece um olhar atento e, eventualmente, estratégias específicas de referenciamento poderiam tornar algumas páginas mais acessíveis e mais bem encontradas na rede da própria Wikipédia.

\section{CONCLUSÃO}

O presente artigo buscou identificar e descrever aspectos da dinâmica social de produção de informações sobre os museus brasileiros na Wikipédia, especificamente dos 20 museus do Ibram que possuem artigos sobre eles. Ao abordarmos características da Wikipédia enquanto plataforma online e aberta à participação do usuário, foi possível observar a relevância e popularidade da Wikipédia enquanto uma fonte de informação online, sendo $05^{\circ}$ site mais acessado do mundo. Esse sucesso da Wikipédia pode ser atribuído ao seu modelo de produção, que ao convidar os usuários a contribuírem com seus conhecimentos acabou por gerar uma dinâmica de participação que permitiu sua escalabilidade e expansão, estando hoje disponível em 303 idiomas, cada um tendo sua comunidade de editores que trabalham para manter os padrões estabelecidos pelas políticas da Wikipédia.

Por meio da coleta de dados do projeto lusófono da Wikipédia, que atende aos países que têm a língua portuguesa como oficial, que é o caso do Brasil, buscamos observar aspectos da produção de informações sobre os museus brasileiros, tomando como amostra os artigos sobre 20 museus do Ibram em edição na Wikipédia.

O dados foram coletados com ajuda do software XTools. Por meio desse software, foi possível obter dados referentes à quantidade de editores e edições por artigo, data de primeiras e últimas edições. Esses dados nos ajudaram a identificar a quanto tempo esses artigos existem, quando foram criados, quantas pessoas participaram em seu processo de edição e quantas vezes foi editado. Também foi possível coletar dados relativos à quantidade de edições por ano e o username dos 10 editores com o maior número de participações por artigo. Com esses dados, foi possível identificar a frequência de participação na edição das páginas ao longo dos anos e identificar a dinâmica de participação dos editores no conjunto dos artigos da amostra. Com base nestes últimos dados, foi possível construir uma rede de relacionamentos que evidencia o grau de participação dos usuários. Dessa forma, identificamos a quantidade de editores responsável por tecer a rede que conecta diferentes artigos sobre os museus do Ibram. E por último, foi possível coletar dados acerca da quantidade de links que entram ou saem dos artigos para outros artigos da Wikipédia, o que nos permitiu observar a dinâmica de referenciamento entre páginas. 
Com base nesse conjunto de dados, foi possível descrever a dinâmica de participação social na construção das informações desse campo.

Os resultados apontam uma rede expressiva de participantes e ações de curadoria ocorrendo de forma transversal entre os diferentes museus. Identifica-se grande desigualdade na dinâmica de produção de informação, ressaltando que os usuários contribuem, em média, com em torno de duas edições por página, fazendo com que os museus com maiores descrições textuais sejam aqueles que receberam contribuições de mais usuários ao longo do tempo.

Dessa forma, por meio do presente estudo foi possível demonstrar as potencialidades da Wikipédia como fonte de consulta de informações sobre o campo museal brasileiro, e também enquanto uma rica fonte de dados que permitem a observação de fenômenos sociais relacionados às interações colaborativas na construção coletiva de conhecimento.

Esperamos que esse trabalho dê subsídios para o desenvolvimento de outros estudos e pesquisas no campo das humanidades digitais e que inspire os profissionais do setor dos museus a participarem dos processos colaborativos de produção de conhecimento sobre o campo museal na Wikipédia, contribuindo assim para uma maior visibilidade dos museus brasileiros na internet e também para a qualificação das informações já disponíveis.

Artigo recebido em 31/01/2019 e aprovado em 07/05/2019.

\section{REFERÊNCIAS}

Berry, D. M. The computational turn: thinking about the digital humanities. Culture Machine, v. 12, p. 1-22. 2011. Disponível em: <https://sro.sussex.ac.uk/49813/1/BERRY_2011THE_COMPUTATIONAL_TURN_THINKING_ABOUT_THE_DIGITAL_HUMANITIES.p.pdf >. Acesso em: $11 \overline{\mathrm{fev}} .2019$.

BLATTMANN, U.; SILVA, F. Colaboração e interação na web 2.0 e biblioteca 2.0. Revista ACB: biblioteconomia em Santa Catarina, Florianópolis, v. 12, n. 2, p. 191- 215, jul./dez. 2007. Disponível em: <https://revista.acbsc.org.br/racb/article/view/530>. Acesso em: 20 dez. 2018.

CAMPELLO, B.; CALDEIRA, Paulo. Introdução às fontes de informação. Belo Horizonte: Autêntica, 2008.

D'ANDREA, C. F. B. Enciclopédias na web 2.0: colaboração e moderação na Wikipédia e Britannica online. Em Questão, v. 15, n. 1, p. 73-88, 2009. Disponível em: <https://seer.ufrgs.br/EmQuestao/article/view/9147/5801>. Acesso em: 20 dez. 2018.

GILES, J. Internet encyclopaedias go head to head: Jimmy Wales' Wikipedia comes close to Britannica in terms of the accuracy of its science entries, a Nature investigation finds. Nature, v. 438, n. 15, dez. 2005. Disponível em: <https://www.nature.com/articles/438900a.pdf>. Acesso em: 20 de dez. 2018.

GONÇALVES, M. Wikipédia: discurso e validade da informação. 2014. Tese (Doutorado em Ciência da Informação ) - Programa de Pós-Graduação em Ciência da Informação, Instituto Brasileiro de Informação em Ciência e Tecnologia/Universidade Federal do Rio de Janeiro, Rio de Janeiro, 2014. Disponível em: <http://ridi.ibict.br/handle/123456789/860>. Acesso em: 20 dez. 2018. 
HJØRLAND, B. Evaluation of an information source illustrated by a case study: effect of screening for breast cancer. Journal of the American Society for Information Science and Technology, v. 62, n. 10, p. 1892-1898, 2011.

KERN, V. A Wikipédia como fonte de informação de referência: avaliação e perspectivas. Perspectivas em Ciência da Informação, v.23, n.1, p.120-143, 2018. Disponível em: <http://portaldeperiodicos.eci.ufmg.br/index.php/pci/article/view/3224>. Acesso em: 20 dez. 2018.

MEDEIROS, J.; SOUZA, R. Informações, fontes, Wikipédia: questões levantadas: apontamentos necessários. P2P \& Inovação, Rio de Janeiro, v.5 n.1, p.70-88, set.-fev. 2018. Disponível em: <http://revista.ibict.br/p2p/article/view/4280/3767>. Acesso em: 20 dez. 2018.

MESGARI, M. et al. "The sum of all human knowledge": a systematic review of scholarly research on the content of Wikipedia. Journal of the Association for Information Science and Technology, v. 66, n. 2, p. 219- 245, 2015. Disponível em:<http://orbit.dtu.dk/files/103083646/WikiLit_Content_open_access_version.pdf>. Acesso em: 20 dez. 2018.

STIVILIA, B. et al. Assessing information quality of a community-based encyclopedia. Proc. IClQ, p.442-454, 2005. Disponível em: <http://mitiq.mit.edu/ICIQ/Documents/IQ\%20Conference\%202005/Papers/AssessingIQ ofaCommunity-basedEncy.pdf $>$. Acesso em: 20 dez. 2018.

WIKIMEDIA FOUNDATION. Wikimedia Foundation mission. 2018. Disponível em: <https://wikimediafoundation.org/about/mission/>. Acesso em: 1 fev. 2019. 